Usi ng questi on words or asking yes/no quest i ons: Fai I ure and success i n cl ar i fying the i nt ent i ons of a boy with hi gh- funct i oni ng aut i sm

\begin{tabular}{|l|l|}
\hline 著者 & O Nanabu \\
\hline $\begin{array}{l}\text { j our nal or } \\
\text { publ i cat i on t i t l e }\end{array}$ & Cl i ni cal Li ngui st i cs and Phonet i cs \\
\hline vol une & 22 \\
\hline nunber & $10-11$ \\
\hline page r ange & $814-823$ \\
\hline year & $2008-10-01$ \\
\hline URL & ht t p: //hdl . handl e. net /2297/19319 \\
\hline
\end{tabular}




\title{
USING QUESTION WORDS OR ASKING YESINO QUESTIONS: FAILURE AND SUCCESS IN CLARIFYING THE INTENTIONS OF A BOY WITH HIGH-FUNCTIONING AUTISM
}

\author{
Manabu Oi Kanazawa University \\ Phone +81-76-264-5512 Fax +81-76-264-5510 \\ e-mail:oimanabu@ed.kanazawa-u.ac.jp
}

\begin{abstract}
This paper examined the processes during which an adult failed to comprehend the intentions of a boy with high-functioning autism. In a dyadic conversation between the adult and the young boy, the adult used questions to elicit clarification of intentions. The boy's responses were examined frame-by-frame using videotape microanalysis. Two causes of adult misunderstanding were the formulaic use of words that confuses the listener and the directional gaze away from the listener. Questions that used interrogatives could easily fail to clarify the intention of the boy and yes/no questions appeared suitable to clarify his intention. However, success or failure to clarify an intention was not based solely on differences in the question format. Differences in question content may also be relevant. Yes/no questions were mainly used to ask about specific actions. On the other hand, interrogative questions were used to handle cognitive or meta-cognitive matters.
\end{abstract}

In conversing with a person who has high-functioning autism (HFA), a non-disabled person will frequently fail to comprehend the communicative intentions of the autistic person. There are two ways to elicit clarification of communicative intentions. One method is to use question words and the other is to use yes/no questions. However, these two methods may vary widely in their ability to elicit meaningful clarification. Oi (2005) showed that two Japanese boys with HFA were unable to make their intentions clear when adult assistants asked for clarification using question words. But they were able to clarify their intentions when the questions were 
changed to a yes/no format.

This difference in efficacy between using question words and using yes/no questions may be common to all languages. For nonecholalic English-speaking autistic children with mild mental retardation, Curcio and Paccia (1987) showed that yes/no questions were more effective in eliciting adequate responses to adult utterances than wh-questions. With Japanese, whose grammar is very different from English, rules for wh-question construction, wh-movement, and auxiliary or substitute verb movement do not exist. English also has strict rules for auxiliary and substitute verb movement in yes/no questions, but not Japanese. Japanese has no substitute verbs while auxiliary verbs join with inflected main verbs.

Thus, the differences mentioned above may have more to do with pragmatic aspects of language than grammatical aspects. Curcio and Paccia suggested that a substantial portion of these children's difficulties as responders in a conversation stems from a failure to process certain information contained in questions from an adult. Therefore, it is necessary to investigate what kinds of information these questions contain to determine what the children are unable to process. To examine this issue, we need to take a close look at a conversation by tracing entire movements from where an adult initiates questions to where a child responds to those questions, step by step from the very beginning of the conversation to the end. Oi's study (2005), which included questions from adults, did not include such a thorough inspection of conversation. It only analysed two brief conversation extracts, each consisting of several conversational turns.

Hewitt (1998) made a study on how well autistic persons comprehend conversational questions. She showed that in young adults with autism, who were native English speakers and functioning in the mild-to-borderline range of mental retardation, four types of questions showed a higher failure rate than the overall failure rate for responses to conversational questions. The four types are questions with more than seven words in length, multi-clauses, inferential requirements, and indirect requests for information. Although the results have no direct relationship with the topic of this paper, failure with these types of 
questions may help explain the difference between wh-questions and yes/no questions.

Tager-Flusberg (1994) studied this difference between wh-questions and yes/no questions from a perspective of production of questions, not comprehension. She looked at form and function relationships in questions produced by English-speaking autistic and Down syndrome children matched for mean length of utterance. She showed that for questions seeking information, agreement, and clarification autistic children with IQs ranging from 61 to 108 rely significantly more on yes/no questions than children with Down syndrome who used wh-questions far more frequently. Combining this result with that of Curcio and Paccia (1987), it seems probable that autistic children have some unique difficulty handling wh-questions. Few clues are available thus far, however, about how and why this problem occurs.

Studies conducted to compare comprehension between yes/no questions and wh-questions in English speaking children who are developing typically depict quite a different picture. Peterson, Dowden, and Tobin (1999) pointed out the increased attention that researchers paid to get accurate information by asking yes/no and wh-questions to preschoolers in police investigations and in courtrooms as witnesses. According to their review of the literature on this subject, although the accuracy of information elicited by means of open-ended questions is typically better than that elicited by more specific questions, most of which are yes/no questions, preschoolers are unlikely to provide very much information without prompting with specific questions. A study by Petersen et al. indicates that yes/no questions frequently elicited errors which were less evident when questions have a wh-format, which on the other hand increased the likelihood of preschoolers saying 'I don't know.' Although the picture depicted here seems to be reversed compared to that hypothesized in children with autism, some risks inherent in yes/no questions should not be overlooked for these children as well.

Focusing on the grammatical aspect of the problem, a study by Deevy and Leonard (2004) on comprehension of wh-questions in English-speaking children with specific language impairment may provide clues to understand characteristics related to the difficulty of handling 
wh-questions for children with autism. They indicated that those children had poorer performance on long object questions compared to long subject questions; and they were less accurate on long object questions than children who were developing typically. Sentence length or degree of grammatical complexity of question may play a significant role in comprehension of wh-questions by children with HFA who generally experience language delay in their early childhood.

This paper examines the processes during which an adult assistant failed to comprehend the intentions of a boy with HFA. In a dyadic conversation between an assistant and a young boy, an assistant used questions to elicit clarification of intentions. The boy's responses were examined frame-by-frame using videotape microanalysis to answer the following questions. 1) What kind of communicative act by the child led to comprehension failure and a question being asked by the assistant? 2) What part of the assistant's question was difficult for the child to respond to? 3) Was there any difference between wh-questions and yes/no questions that influenced the child's ability to respond? 4) Did sentence length or grammatical complexity influence a child's ability to respond?

\section{Participants}

A native Japanese boy, D, diagnosed with HFA participated in this research. D was 6 years old and his IQ was 90 as measured by WISC-III . D's assistant was a graduate student, $R$ in her early twenties, who majored in communication disorders.

\section{Data collection}

One extract was selected from dyadic conversation as follows. First, the assistant freely selected a target situation on the video (the total length of the conversation was about ten minutes) in which the assistant herself thought that the conversation had broken down when asking questions to the child. Conversational breakdown was defined as a situation in which the assistant thought that she had failed to clarify the child's intention. Second, the target situation was traced backwards until the topic of the 
situation occurred for the first time. The conversation extract was defined as the period from the initiation of the topic to the completion of the topic conversation flow, including the breakdown, at the point where the conversation shifted to the next topic. A rough transcription of the extract made by the assistant was refined and analysed jointly with the author while comparing the transcripts with the video recording.

\section{Analysis}

For analysis, the author cooperated closely with the assistant to clarify the conversational flow, turn by turn, for what was intended or assumed by the speaker for each utterance and gesture, for how the utterances and the gestures were interpreted by the listener, and for what kind of impact the interpretation had on the listener. For questions asked by the assistant, the author asked why she asked each question and what kind of response she expected from each child.

This kind of detailed analysis attempts to shed light on how a conversation emerges beyond merely tracing conversational 'footprints in the sand' (Clark, 1996:337). The aim of reconstructing how the assistant and the child walk together or collide with each other in conversation is to describe the underlying processes from which an assistant's question arises and ultimately succeeds or fails to clarify the child's intention.

\section{Conversation extract}

Below is a description of a conversation that occurs between $D$ and assistant $R$. The conversation takes place in the university playroom where the two play a game of bowling together for the first time. The object of the game is to use a rubber ball to topple plastic pins that have been lined up. This conversation extract begins right after D's declaration 'Omedeto, boku no kachi!'(Congratulations! I'm the winner!) that he utters after winning two times out of three.

1D: Chtto kono tokorode $=((\mathrm{D}$ stands while raising the ball over his head. Next he dribbles the ball, following it with his eyes.)) = mata ganbareba Hold it there It'd be good I: kedo. ((D dribbles the ball some more)) Mou game ha oshimai nanda 
to try again, but.

The game is all over now,

kedo. ( $\mathrm{D}$ raises the ball over his head with both hands and looks in front of him.))

but.

2R: Mo: bowling oshimai? ((R peeks at D's face from the side.))

Is bowling all over now?

3D: Un. ((While sticking out the ball in front of his body, D says the following with a blank expression.)) Oshimai nanda kedo

Uh-huh. It's all over, but.

4R: Oshimai nanda kedo, na:ni?

What do you mean, It's all over, but?

5D: Mo: game ha oshimai nanda kedo. ((Taking the ball, D turns his back to R.)) Oshimai, oshimai nanda kedo, oshimai. ((D walks toward the toy shelf.))

The game is all over now, but. It's all over. It's all over, but. It's all over.

6R: D, atama fuko: yo. ((R holds out a towel to D.))

$D$, wipe your head.

7D: Ja: $=((\mathrm{D}$ looks at himself in a big mirror as he wipes away the sweat. $))$

Okay then

= ichinichi, mo: ichido ganbareba $i$ : yo ne. ((D raises his right hand.))

one day, it'd be good to try again, right?

Kyou ha mou kore de oshimai. ((D joins his left hand with his raised right hand.))

This is all over for today.

8R: Bowlong oshimai?(( $\mathrm{R}$ points at the pins.)) [Kore de]

Bowling is all over? [This]

9D:((D steps toward R. $))[U n]=((D$ nods and looks at R. $))=$ Kyou ha mo: [Uh-huh] For today

kore de oshimai.

this is all over.

10R: Kore dou siyou?

((R points at the pins again.)) What about these?

11D: Un, chotto,

ja: tugini okataduke wo goshoukai 
Uh-huh, just a, ((D looks at the pins.)) well then, next let's introduce Itadaki mashou.

$\mathrm{Ha}$ :

tidying up. ((D faces and walks toward the mirror.)) Aah ((Sighs.))

12R: Okataduke suru no?

Tidying up?

13D: Ja: mo: ichido ganbareba i: no desu.

Okay then it'd be good to try it again. ((D turns and walks toward R.)) 14R: Mo: ikkai bowling suru no?

Do you want to play bowling one more time?

15D: Un.

Dakara

taoreta tokoro

Uh-huh. $((D$ nods. $))$ So $=((D$ points at the pins. $))=$ the toppled pins de mata motodouri zero ni naru are set up like before. ((After uttering while facing the mirror, D turns and faces R.)) Jaa motodouri de hayaku pin wo taoseta hou ga kachi da yo.

Okay then the first one to knock down the pins is the winner. All Yosi kondo koso, ma, ganbatte ne.

right, come on, give it a try. ((D raises his right hand.)) 16R:Un.

Uh-huh.

17D: Deha mata ne :. ((D waves his right hand.))

See you again.

18R: Deha mata ne:? ((R laughs.))

See you again?

19D: Jaa bai bai shite kara bowling siyou ne. ((D places both hands on his waist and turns toward the mirror.))

Okay then after bye-bye let's play bowling.

20R: Un.

Uh-huh.

21D: Bai ba::I.((D turns around while looking at the mirror.))

Bye-b::ye.

Ja: bowling siyou ((While continuing to look at the mirror, after waving both hands D looks toward R.))

Okay then let's play bowling.

In turns 1 and 3 , D wants to break off play at an advantageous point to 
ensure he is the champion. Midway through the conversation extract, he changes his mind. Finally, in turn 21 he proposes a resumption of play. $\mathrm{R}$ has difficulty understanding D's intentions, so she asks questions in seven of her 10 conversation turns. Two questions use interrogatives and the other five are yes/no questions. What follows is a background description of each question and whether D's response is appropriate.

The first question $R$ asks $D$ is in turn 2. It is a yes/no question that prompts $D$ to make a decision. Part of D's utterance is repetitive with a rising intonation. $R$ gives two reasons for asking a question like this. Firstly, the half-finished and roundabout use of the word 'but' (nanda kedo) is an obstacle to a clear understanding of D's intentions. $R$ did not know whether $\mathrm{D}$ wanted to stop the bowling game or had something else in mind. Secondly, D spoke, as if to himself, without turning to face R. So R had some doubt as to whether D's utterance about ending the game was really what he wanted to do. Because of these two factors, R was not able to get a strong sense that it was time to take steps to end the game. D shows two reactions in turn 3 in response to the question in turn 2. Firstly, D gives an affirmative response to R's prompt to make a decision. Secondly, however, $\mathrm{D}$ again repeats the roundabout utterance 'It's all over, but' (oshimai nanda kedo). As in turn 1, D does not look toward R.

In turn 4, R's question uses an interrogative. The reason for this question is that $R$ wants $D$ to fill in the information seemingly omitted by using the word 'but' (kedo) in turn 3. R also wants to find out the real reason why $D$ repeatedly uses this roundabout utterance. In response to R's question in turn 4 , in turn $5 \mathrm{D}$ merely repeats an even shorter phrase 'It's all over' (oshimai) without supplying any of the words seemingly omitted by using the word 'but' (kedo). As if trying to escape the task of clarifying himself, D moves away from R. In turn 5, D seems like he wants to respond; but here we take the interrogative question as a clue to suggest he finds it difficult to add further to the conversation. By moving away from $R$, he can escape this difficulty and end the conversation.

R's next question appears in turn 8. As in turn 2, it is a yes/no question that prompts $D$ to make a decision. According to $R$, she has three reasons for asking $D$ to clarify his intentions again. Firstly, $R$ does not understand D's second utterance in turn 7, 'one day, it'd be good to try again, right?' 
(ichinichi, mo: ichido ganbareba $i$ : yo ne). Secondly, R is confused by the manner in which $D$ moves in front of the mirror and speaks as if to himself. Thirdly, R does not understand D's gesture in front of the mirror in which he raises and joins his hands together. In turn 9, $D$ gives a clear affirmative response to R's prompt to make a decision. At this time, it looks like $R$ and $D$ both agree that the bowling game has come to an end.

After receiving this response from $D$, in turn $10 R$ asks $D$ an interrogative question related to tidying up the scattered pins after the bowling game. What $R$ wants to find out from this question is whether $D$ himself intends to pick up the scattered pins. However, R's question is indirect, vaguely worded, and relies on a few tacit assumptions. One assumption is that after bowling is finished, the pins must be put back in their box. $R$ expects $D$ to understand this implied condition through the conversation flow from turn 9 to 10 . A second assumption is that $R$ and $D$ will pick up the pins together. After each of the three games, $\mathrm{R}$ always reset the scattered pins by herself. While resetting the pins, $D$ would merely drift about with no intention of helping out, which caused $\mathrm{R}$ to become dissatisfied with $D$.

$D$ responds to R's interrogative question about a plan of action. But it is not the response $\mathrm{R}$ expects; namely, one that clarifies his intention to help put away the pins. In addition, D's response takes no account of the tacit assumptions embedded in R's question. For D this second interrogative question presents a situation different from the first interrogative question in turn 4. He thus finds it difficult to handle. D's difficulty becomes apparent in turn 11 in which he says haltingly, 'Uh-huh, just a' (un chotto) and follows this with a sigh, 'aah.' We can infer the meaning of 'aah' by comparing turns 9 and 13 . In turn 9, D clearly states that the bowling game has ended. In turn 13, he utters words about continuing the bowling game. Between turns 9 and 13, D undergoes a change of mind from ending the game to continuing it. D's sigh expresses his ineffectiveness at communicating what he thinks about ending the game. So we can say that R's interrogative question has the effect of making D change his mind. D's change of mind is based on misunderstanding R's intention in turn 10. This misunderstanding is due to R's vaguely worded question that contains tacit assumptions. Another way to look at it, D is not able to understand 
the connotations embedded in turn 10. R wants to ask about D's plan of action related to putting the pins back in the box. But $D$ responds with $a$ plan of action related to resetting the pins. Tracing turns 13, 15, 19, and 21 , we can see that D's 'tidying up' (okatazuke) does not mean putting the pins back in the box, which in turn 10 is an act the assistant assumes will happen. It becomes clear 'tidying up' means resetting the pins to an upright position. In addition, it is likely $D$ thinks that $R$ is the person who will perform the action in response to 'Let's introduce tidying up.' In fact, after this conversation extract, $R$ neatly reset the toppled pins while $D$ stood by idly.

R's question in turn 12 is a result of D's strange utterance in turn 11 . R thinks D's 'tidying up' (okatazuke) means to put the pins back in the box; but she is not confident whether she understands this odd expression. The expression 'Let's introduce tidying up' (okatazuke wo goshokai itadaki masho) is not incorrect grammatically, but it is inappropriate. People who participate in some event at a certain place would expect the object of the phrase 'let's introduce' to be a new or unfamiliar person or thing. However, for the object $D$ uses a noun that expresses an action. The phrase 'Well then, next let's introduce _ _ ' is a line often used by television program MCs. Normally an MC uses this expression to introduce a novel person, product, or place of interest to a special panel of guests and the viewing audience. The expression uses a polite form of address suitable for an audience. Turn 11 is an example of a formulaic utterance (Dobbinson, Perkins, and Boucher, 2003) in which an autistic person borrows a familiar expression, in this case a standard line used by TV program MCs. In this playroom setting, we can think of $D$ as the $M C$ and $R$ as a special panel guest.

This utterance has other oddities as well. Firstly, in Japanese the form of 'tidying up' (okatazuke) is an expression that an adult would use to a child, who in turn would use it by way of imitation. Secondly, D uses a mature adult way to say 'let's introduce' (goshokai itadaki masho), which incorporates two very polite forms 'go' and 'itadaki.' So 'Let's introduce tidying up' combines two phrases that belong in completely different language registers, which is what gives the utterance its unique strangeness. 
Thirdly, in turn 11 it is strange how D walks toward the mirror, talks in front of it, then lets out a sigh. D also speaks and gesticulates in front of the mirror before in turn 7 and after in turns 15, 19, and 21. These utterances, including the times in which he is not facing the mirror, are all very formulaic. His speaking tone and style sound like they are modeled after someone. D's formulaic utterances may be related to iconic memory, which is awakened by looking into the mirror, resulting in verbal reproduction. This chain of oddities in turn 11 confuses $R$ who in turn 12 asks $D$ a yes/no question for clarification. D's response in turn 13 is not a direct yes or no, but it relates closely to the matter $R$ is trying to communicate. As stated above, $R$ and $D$ likely mean two different things by 'tidying up' (okatazuke). D interprets turn 12 as a question that asks whether the pins should be reset so the bowling game can continue. Turn 12 serves as a confirmation of D's message in turn 11 and as a step closer to a resumption of play. It also links with D's utterance in turn 13 'It'd be good to try again.' (mo: ichido ganbareba $i$ :) .

In turn 14, $\mathrm{R}$ has three reasons to use another yes/no question to prompt $D$ to make a decision. Firstly, $R$ feels that $D$ 's utterance in turn 13 to resume bowling is abrupt. In turns 8,10 , and 12, R's utterances move consistently toward ending the game; and she interprets D's utterances in turns 9 and 11 as moving in the same direction. Secondly, D's utterance does not respond directly to R's question. 'It'd be good to try' (ganbareba $i$ ) refers to bowling matters. Though D's utterance apparently relates to turn $12, \mathrm{R}$ is unable to understand the connection. Thirdly, the expression ' $i$ : no desu,' like the formal usage in turn 11 , is unnecessarily polite. In turn 15 , D gives a direct affirmative reply to R's yes/no question for clarification. But that's not all. $D$ explains the rules of the game and cheers on $R$ who is the loser thus far. This utterance is also an easy one for $R$ to understand.

In turn 18, R asks another yes/no question. The falling intonation contour of D's utterance in turn 17 is changed to a rising contour by $R$ in turn 18. This shows that $R$ fails to understand D's illocutionary intention and that $R$ indirectly expects $D$ to clarify his intentions. R's question along with her subsequent laugh also expresses how she has become baffled by D's utterance in turn 17. She cannot understand the connection between D's utterances in turns 15 and 17. In addition, R can imagine neither to 
whom the parting greeting is intended nor the timing. R's question in turn 18 is an attempt to resolve this baffling situation. However, D's response in turn 19 ignores her question. D gives appropriate responses to R's four previous yes/no questions, but not to this one. There may be two reasons for this. Firstly, D may not be able to respond to R's indirect question about the intended meaning of the utterance. Secondly, D may not notice R's baffled expression or he may have a problem with emotional reaction and expression.

The morpheme length of utterance in R's five yes/no questions ranges from two to five and that in the two questions that use interrogatives is four and three respectively. So the morpheme length is similar for both types of questions.

\section{Discussion}

Our analysis of conversation extract 1 suggests that D's communicative acts have characteristics that lead $\mathrm{R}$ to ask certain questions. These characteristics include the use of formulaic and inappropriate words and speaker's directional gaze. The former obscures the illocutionary intention, which makes $R$ ask a question to clarify D's intention. The latter casts doubt on the sincerity of the speaker. This makes $R$ ask questions to discover whether $D$ is in the mood to communicate.

R's questions are divided into ones that are easy for $D$ to respond to and ones that are difficult. Questions that present no problem are ones such as 'All over?', 'Tidying up?', and 'Do you want to play bowling?' that ask D whether he intends to do a specific action. These easy questions are ones that can be answered with a yes or no. Four out of the five times R asks this type of question, she obtains an appropriate response from $D$. In contrast, D has difficulty responding to the two interrogatives. However, in each case the reason for difficulty is different. The difficulty in turn 4 is the way in which $\mathrm{R}$ prompts $\mathrm{D}$ to clarify his illocutionary intention and to take cognitive action. The difficulty in turn 10 may be due to one or both of the following: the tacit assumptions underlying the question or the implied meaning produced in the question based on the response in turn 9.

There are two reasons why the yes/no question in turn 18 is difficult compared to four others of the same type. Firstly, turn 18 relates to 
cognitive matters by indirectly inquiring about the intention in D's utterance. It is not like the four other yes/no questions that directly inquire about the intention of specific actions. Secondly, $\mathrm{R}$ reveals an emotional state of confusion that is absent in the four other questions.

Comparatively speaking, it appears that $D$ can handle yes/no questions more easily than interrogative questions. But as seen in turn 18, depending on the content, yes/no questions may also present difficulties.

Our analysis exposes the fine details of the conversation extract and suggests answers to four questions related to our research.

The first question asks whether some kind of communicative act by a child can cause an adult to misunderstand that child's intention. Two causes of adult misunderstanding are the formulaic use of words that confuses the listener and the directional gaze away from the listener.

Answers to the second and third questions are closely connected, so they are grouped together. Can a child respond appropriately to an adult's question and successfully make his intentions clear? To a large degree, it depends on whether the question uses an interrogative or a yes/no format. However, it is also relevant to consider differences in information characteristics among questions of the same type. The interrogative questions that fail to clarify intentions call on the child to handle cognitive or meta-cognitive information such as 1) to clarify the child's own illocutionary intentions, 2) to take account of tacit assumptions in an adult question, 3) to explain the child's own plan of action. In contrast, most yes/no questions that successfully clarify intentions ask whether the child intends to do a specific action. However, $D$ is unable to respond to this type of question when he is asked to clarify his illocutionary intentions.

There is a clear difference between how easy it is to respond to each type of question. It is also obvious that this difference is related to the information characteristics of the question such as whether the content is cognitive or behavioral.

The fourth question asks whether the two types of questions differ in sentence length and whether yes/no questions look longer. To judge from just looking, we do not think a child's difficulty in responding to a question is related to the sentence length of the question.

For yes/no questions, out of five questions for $D$, four questions that ask 
whether a child intends to do a specific action obtain either an affirmative response or one in compliance with the adult prompt, and the other one question obtains a response that ignores the adult question. None of these questions obtain a negative response. The findings here are similar to Peterson et al. (1999) who expose a bias in favor of affirmative responses in yes/no questions. However, our findings do not affirm false facts as shown in Peterson et al. Our content shows how a child expresses his intention to do an action. Although it is possible for a child with autism to give an affirmative response that is contrary to his intention or the facts, the yes/no questions taken up in our research offer no positive proof of such a risk.

Two conclusions suggested by this research are 1) questions that use interrogatives can easily fail to clarify the intention of a child with autism and 2) yes/no questions appear suitable to clarify the intention of a child with autism. However, success or failure to clarify an intention is not based solely on differences in the question format. Differences in question content may also be relevant. Yes/no questions are mainly used to ask about specific actions. On the other hand, interrogative questions are used to handle cognitive or meta-cognitive matters. For an inquiry about a specific action, an interrogative question may be able to clarify what a child intends to do. And for an inquiry about a cognitive matter, a yes/no question may also not be able to obtain clarification. To discover more about these types of questions, we need to obtain relevant conversation data and move further ahead with our research.

\section{References}

Clark, H, H. (1996): Using language. Cambridge University Press.

Curcio F. and Paccia J. (1987): Conversations with autistic children: contingent relationships between features of adult input and Children's response adequacy. Journal of Autism and Developmental Disorders, 17, 1, 81-93.

Deevy, P. and Leonard, L.B. (2004): The comprehension of wh- questions in children with specific language impairment. Journal of Speech, Language, and Hearing Research. 47,802-815.

Dobbinson, S., Perkins, M., and Boucher, J. (2003): The interactional 
significance of formulas in autistic language. Clinical Linguistics and Phonetics, 17, 4-5,299-307

Hewitt, L E. (1998): Influence of question type on response adequacy in young adults with autism. Journal of Communication Disorders 31,135-152.

Oi, M. (2005): Interpersonal Compensation for Pragmatic Impairments in Japanese Children with Asperger Syndrome or High-Functioning Autism.

Journal of Multilingual Communication Disorders, 3, 3,203-210.

Peterson, C., Dowden, C., and Tobin, J. (1999): Interviewing preschoolers: Comparisons of yes/no and Wh- questions. Law and Human Behavior, 23, 5,539-555

Tager-Flusberg, H. (1994) Dissociation in form and function in the acquisition of language by autistic children. In: H. Tager-Flusberg (Ed.) Constraints on language acquisition: Studies of atypical children, (pp161-172). Lawrence Erlbaum, Hillsdale, New Jersy.

Appendix: key to transcription symbols in Japanese conversation text

'[' and ']' A left bracket links an ongoing utterance with an overlapping utterance at the point where the overlap begins and a right bracket links at the point where the overlap ends.

'=‘ An equal sign marks where there is no interval between adjacent words or utterances.

'?' A question mark indicates the end word has rising intonation contour.

$\because$ A period indicates the end word has a falling intonation contour.

$\because$ A comma indicates a continuing intonation.

' $\because$ A colon indicates an extension of the sound or syllable it follows.

'(())' Text in double brackets indicates a gloss or description of a nonverbal aspect of the conversation. 